MS39 X-Ray diffraction on the

$\mu$ s to ps time scale

Chairs: Semën Gorfman, Michael Wulff

\section{MS39-P1 Time-Resolved Crystallography \\ from femtoseconds to microseconds at the \\ laser driven X-ray plasma source in ELI beamline}

Borislav Angelov ${ }^{1}$, Jakob Andreasson ${ }^{1}$

1. Institute of Physics, ELI Beamlines, Academy of Sciences of the Czech Republic, Na Slovance 1999/2, CZ-18221 Prague, Czech Republic

email: borislav.angelov@eli-beams.eu

The novel laser driven plasma X-ray sources will allow for time resolved X-ray scattering and diffraction on a femtosecond to microsecond time scale. Unlike large scale facilities such as synchrotrons, intensive lasers can be used for the generation of short X-rays pulses in a setup of much smaller size that will be suitable for implementation in a laboratory. The femtosecond laser driven emission of X-ray pulses from plasma (XPS) offers higher time resolution for fast kinetic measurements than continuously emitting sources. The ELI beamlines facility is planned to start operation by the end of 2016 in Dolni Brezany, Czech Republic. The first users are planned for 2018. ELI beamlines will give a unique advantage for time resolved crystallography and wide angle scattering from crystalline samples, including proteins. The generated pulses will span approx. $100 \mathrm{fs}$ with a repetition rate of $1 \mathrm{kHz}$. The intensity of the X-ray pulses on the sample will be $\sim 10^{\wedge} 6 \mathrm{ph} /$ pulse at $10.8 \mathrm{KeV}$. The scattered and diffracted by the crystal X-rays will be counted using a DECTRIS Eiger $1 \mathrm{M}$ area detector which operates at the same frame rate as the source, i.e. $1 \mathrm{kHz}$. Such setup can be combined with several pump probe lasers to study the fast kinetics for example in proteins relevant to plant photosystems or vision in animals. To obtain a representative statistics from the measurements, it will be necessary several repetitions to be done. Because of the interdisciplinary nature of the fields and of the ELI beamlines facility regular discussions between experts in the field of high power laser-matter interaction and potential users, as well as young scientists, are organized.

References: 1. B. Rus ; F. Batysta ; J. Čáp ; M. Divoký ; M. Fibrich, et al. "Outline of the ELI-Beamlines facility", Proc. SPIE 8080, Diode-Pumped High Energy and High Power Lasers; ELI: Ultrarelativistic Laser-Matter Interactions and Petawatt Photonics; and HiPER: the European Pathway to Laser Energy, 808010, 2011; doi:10.1117/12.890392

Keywords: laser plasma X-rays, diffraction, fast kinetics, Eiger detector
MS39-P2 Time-resolved Local Dynamics

Measurement of Supersaturated Solution by Diffracted X-ray Tracking

Yufuku Matsushita ${ }^{1}$

1. The University of Tokyo, Graduate School of Frontier Sciences, Kashiwa, Japan

email: 8113178469@mail.ecc.u-tokyo.ac.jp

Recently, the micro scale structure of the supersaturated solution are receiving a lot of attention in nucleation and crystal growth process of inorganic, organic and protein compounds. In particular, the crystallization and nucleation process from supersaturated solution is known as a local phenomenon and instantaneous process. The observation of supersaturated solution is required a local structure and time resolved behaviour[1]. In this study, we approached to observe the time resolved micro scale dynamics of protein solution by Diffracted X-ray Tracking (DXT) as known as single molecule measurement method. This method is able to direct observation of local solution dynamics for Target solution by detecting angular rotational displacement of a single gold nanocrystal (approximately $100 \mathrm{~nm}$ ) with pico-meter scale positional accuracy and micro-second time resolution. For target sample, we choose a inorganic (Sodium acetate) and Protein supersaturated solution (Lysozyme). From detailed DXT analysis, we observed the crystal precursor state solution both of inorganic and protein compounds are containing slow and fast dynamics, In particular the fast diffusion corresponded relaxation process of supersaturated state of nano scale clusters which is a important factor of maintenance of metastable supersaturated condition, driving force of morphology determination of final product from crystallization and nucleation process from supersaturation. And the force-field values of this diffusion behaviour are detected as $10^{-15}$ and $10^{-18}$ Newton scale in inorganic and protein supersaturated solution, respectively. From this study, DXT measurement results and detailed analysis process for a protein solution such as crystal precursor metastable state of solution are concerned that this technique is a powerful tool for observing nano-scale molecular dynamical structures and its local dynamics in bulk solution. For next work, we will planning to tackle research in order to determine the morphology control factor of final products and inhibit techniques of nucleation by DXT, and instantaneous observation of nucleation process by Pump-and probe measurement.

[1] Y. Matsushita, H. Sekiguchi, K. Ichiyanagi, N. Ohta, K. Ikezaki, Y. Goto, and Y. C. Sasaki, "Time-resolved X-ray Tracking of Expansion and Compression Dynamics in Supersaturating," Nat. Publ. $G r .$, no. November, pp. 1-8, 2015.

Keywords: Supersaturated solution, Diffracted X-ray Tracking 